\title{
Pathways to personalized treatment strategies for depressive disorders
}

\author{
Dorota M. Badowska • Daniela Reich-Erkelenz • \\ Andrea Schmitt $\cdot$ Peter Falkai
}

Published online: 21 December 2014

(C) Springer-Verlag Berlin Heidelberg 2014

In this issue, Möller et al. [1] present the first part of their two-piece invited review on the changes with focus on some general aspects plus specifically depressive disorder introduced in the new DSM-5 classification system. The originally planned introduction of a primarily dimensional diagnostic system in DSM-5 could not stand-up to the categorical system of disorders. Also retained was the symptom-based, descriptive approach at the cost of a more pronounced neurobiological approach including biological markers in favour of more objective psychiatric diagnoses. Accordingly, only a minimal version of a dimensional approach expanded to DSM-5 by introducing several transnosological specifiers plus an option for symptom- or syndrome-related severity and dimensional assessments. Anyhow, some important changes in criteria were made, e.g. in schizophrenia as well as separating depressive and bipolar disorders: DSM-5 at present is the only comprehensive diagnostic manual fully separating depressive disorders from each other by eliminating the comprehensive category of affective disorders, regarding bipolar disorder as a "bridge" between schizophrenia and depressive

\section{M. Badowska}

Department of Neurogenetics, Max Planck Institute

for Experimental Medicine, Hermann-Rein-Straße 3,

37075 Göttingen, Germany

D. Reich-Erkelenz

Institute of Psychiatric Phenomics and Genomics,

Ludwig-Maximilians-University Munich, Nußbaumstr. 7,

80336 Munich, Germany

A. Schmitt $(\bowtie) \cdot$ P. Falkai

Department of Psychiatry and Psychotherapy,

Ludwig-Maximilians-University Munich, Nußbaumstr. 7, 80336 Munich, Germany

e-mail: Andrea.Schmitt@med.uni-muenchen.de disorder, and redefining the boundaries of major depressive disorder. Additionally, new diagnostic categories like the premenstrual dysphoric disorder were introduced. Despite the above named criticisms on DSM-5, the authors expect a significant improvement in certain aspects of diagnosis which will allow a more individualized description of the psychopathological state.

In Europe, burnout is an increasingly favoured concept commonly thought to be triggered by work strain and subsequently itself triggering mental disorder, but a cross sectional study by Rössler et al. [2] points out the importance of individual traits in the development of the burnout syndrome. They report that a history of lifetime mood disorders-especially in combination with anxiety disordersor partnership problems is predictors for burnout. The authors thus query the consideration of mental disorder as occupational illness, since work-related predictors appear to be less prominent than the aforementioned. To examine burnout-depression distinction, Bianchi and Laurent [3] performed an eye-tracking study on emotional information processing in a sample of 54 human service employees. They detected burnout to be-similar to depression-associated with increased attention for dysphoric stimuli and decreased attention for positive stimuli. Because subsequent hierarchical multiple regression analyses revealed a probable interchangeability between burnout and depression, the authors assume structural similarities between both entities and question the singularity of the burnout syndrome. In the modern working environment, high fluid IQ is a demand for many challenges. In healthy probands, Friedel et al. [4] applied functional magnetic resonance imaging (fMRI) to investigate the relationship between stressful life events, fluid IQ as a measure of cognitive flexibility and learning signals measured as BOLD prediction error signal in ventral striatum. They report that stress 
affected learning strategies by reducing fluid IQ, which correlated with changes in ventrostriatal BOLD signal. Thus, socio-emotional factors such as chronic stress may have impact on fluid IQ and performance.

Since emotional anticipation is a core skill in everyday life and effective cognitive control strategies are neurobiologically represented in prefrontal structures regulating limbic regions, Opialla et al. [5] via fMRI compared the neurobiological mechanisms of cognitive strategies and mindfulness-associated methods, which both are increasingly applied in psychotherapy to improve emotional control. Fifty-three healthy subjects were examined during the cued expectation and perception of negative and potentially negative emotional pictures. The authors found both methods to comparably activate the medial prefrontal cortex and the amygdala. However, the mindfulness strategy induced stronger activation of ventro- and dorsolateral prefrontal areas, supramarginal gyrus, and insula during stimulus expectation of negative versus neutral stimuli, while the perception of these stimuli, again, caused increased activity only in the caudate of the cognitive group. Both strategies recruited overlapping brain regions known to be involved in regulation of emotions. Unravelling the neurobiological background of these alterations may help to elucidate mechanisms underlying psychotherapeutic interventions. Depression is amongst others associated with the inability to properly experience or respond to rewards, which possibly might be due to abnormal modulatory mechanisms. Using a reward fMRI paradigm, Goya-Maldonado et al. [6] identified two types of depressive patients by means of higher or lower activating the nucleus accumbens (NAcc), a region crucially involved in reward processing. Patients with lower NAcc activation confirmed impairments in the bottom-up input by presenting significant hypoactivation in the ventral tegmental area plus bilateral ventral striatum, whereas higher NAcc activation led to significant hyperactivation in prefrontal areas, which might bespeak a disturbed top-down regulation of the NAcc. Thus, the midbrain and prefrontal regions might be specific pathophysiological substrates for these depression subtypes and identifying the two mechanisms with either bottom-up or top-down dysfunctions may have important implications for developing more personalized therapeutic strategies of depression.

Due to the frequent association of thyroid diseases with psychiatric disorders, Degner et al. [7] in a clinical study investigated the comorbidity between autoimmune thyroiditis and depression. They found elevated levels of auto-antibodies against thyroidal peroxides (anti-TPO), a diagnostic feature of autoimmune thyroiditis, in uni- and bipolar depressive outpatients, but no such changes in schizophrenic patients. Therefore, a rather disease-specific association between autoimmune thyroiditis and depression can be assumed, although these results should be confirmed in larger studies, which also applies to a putative physiological background.

Early response to medication against major depressive disorder and reduction in EEG-derived prefrontal theta cordance value enables clinicians to better predict long-term efficacy of antidepressant treatment. In a naturalistic study, Bares at el. [8] analysed early response to medication in 87 patients with major depressive disorder and assessed whether a combination of predictors yields stronger predictive power than an over $20 \%$ reduction in depression score or decrease in prefrontal theta cordance alone. In fact, combined analysis of MADRS scores and prefrontal EEG recordings had much better predictive ability to differentiate responding from non-responding patients. Major depressive disorder and bipolar disorder can be easily misdiagnosed, resulting in improper medication and even enhancement of the symptoms. It is known that brain-derived neurotrophic factor (BDNF) levels are reduced in drug-naïve patients with major depression and restored after treatment with antidepressants. In the letter to the editor, Hashimoto [9] describes recent studies showing that the ratio of proBDNF to mature BDNF in blood may be reduced in major depression and be elevated in bipolar disorder, yet total BDNF is reduced. This leads to the recommendation to assess both forms as probable biomarkers in affective disorders. However, the genetic background (e.g. Val66Met polymorphism), medication, and epigenetic factors have to be taken into account.

\section{References}

1. Möller H-J, Bandelow B, Bauer M, Hampel H, Herpertz SC, Soyka M, Barnikol UB, Lista S, Severus E, Maier W (2014) DSM-5 reviewed from different angles: goal attainment, rationality, use of evidence, consequences-part 1: general aspects and paradigmatic discussion of depressive disorders. Eur Arch Psychiatry Clin Neurosci. doi:10.1007/s00406-014-0520-x

2. Rossler W, Hengartner MP, Ajdacic-Gross V, Angst J (2014) Predictors of burnout: results from a prospective community study. Eur Arch Psychiatry Clin Neurosci. doi:10.1007/ s00406-014-0512-x

3. Bianchi R, Laurent E (2014) Emotional information processing in depression and burnout: an eye-tracking study. Eur Arch Psychiatry Clin Neurosci. doi:10.1007/s00406-014-0549-x

4. Friedel E, Schlagenhauf F, Beck A, Dolan RJ, Huys QJM, Rapp MA, Heinz A (2014) The effects of life stress and neural learning signals on fluid intelligence. Eur Arch Psychiatry Clin Neurosci. doi:10.1007/s00406-014-0519-3

5. Opialla S, Lutz J, Scherpiet S, Hittmeyer A, Jäncke L, Rufer M, Grosse Holtforth M, Herwig U, Bruhl AB (2014) Neural circuits of emotion regulation: a comparison of mindfulness-based and cognitive reappraisal strategies. Eur Arch Psychiatry Clin Neurosci. doi: $10.1007 / \mathrm{s} 00406-014-0510-\mathrm{z}$

6. Goya-Maldonado R, Weber K, Trost S, Diekhof E, Keil M, Dechent P, Gruber O (2014) Dissociating pathomechanisms of depression with fMRI: bottom-up or top-down dysfunctions of the 
reward system. Eur Arch Psychiatry Clin Neurosci. doi:10.1007/ s00406-014-0552-2

7. Degner D, Haust M, Meller J, Ruther E, Reulbach U (2014) Association between autoimmune thyroiditis and depressive disorder in psychiatric outpatients. Eur Arch Psychiatry Clin Neurosci. doi:10.1007/s00406-014-0529-1

8. Bares M, Novak T, Kopecek M, Brunovsky M, Stopkova P, Hoschl C (2014) The effectiveness of prefrontal theta cordance and early reduction of depressive symptoms in the prediction of antidepressant treatment outcome in patients with resistant depression: analysis of naturalistic data. Eur Arch Psychiatry Clin Neurosci. doi:10.1007/s00406-014-0506-8

9. Hashimoto K (2014) Brain-derived neurotrophic factor (BDNF) and its precursor proBDNF as diagnostic biomarkers for major depressive disorder and bipolar disorder. Eur Arch Psychiatry Clin Neurosci. doi:10.1007/s00406-014-0557-x 